\title{
Evaluations for abuse in young children with subdural hemorrhages: findings based on symptom severity and benign enlargement of the subarachnoid spaces
}

\author{
Jennifer B. Hansen, MD, ${ }^{1}$ Terra Frazier, DO, ${ }^{1}$ Mary Moffatt, MD, ${ }^{1}$ Timothy Zinkus, MD, ${ }^{2}$ and \\ James D. Anderst, MD, MSCI ${ }^{1}$
}

Departments of ${ }^{1}$ Pediatrics and ${ }^{2}$ Radiology, Children's Mercy Hospital, Kansas City, Missouri

OBJECTIVE Children who have subdural hematomas (SDHs) with no or minimal neurological symptoms (SDH-mild symptoms) often present a forensic challenge. Nonabusive causes of SDH, including birth-related SDH, benign enlargement of the subarachnoid spaces (BESS), and other proposed causes have been offered as etiologies. These alternative causes do not provide explanations for concomitant suspicious injuries (CSIs). If SDH with mild symptoms in young children are frequently caused by these alternative causes, children with SDH-mild symptoms should be more likely to have no other CSIs than those who have SDH with severe symptoms (SDH-severe symptoms). Additionally, if SDH with mild symptoms is caused by something other than abuse, the location and distribution of the SDH may be different than an SDH caused by abuse. The objectives of this study were to determine the prevalence of other CSIs in patients who present with SDH-mild symptoms and to compare that prevalence to patients with SDH-severe symptoms. Additionally, this study sought to compare the locations and distributions of SDH between the two groups. Finally, given the data supporting BESS as a potential cause of SDH in young children, the authors sought to evaluate the associations of BESS with SDH-mild symptoms and with other CSIs.

METHODS The authors performed a 5-year retrospective case-control study of patients younger than 2 years of age with SDH evaluated by a Child Abuse Pediatrics program. Patients were classified as having SDH-mild symptoms (cases) or SDH-severe symptoms (controls). The two groups were compared for the prevalence of other CSIs. Additionally, the locations and distribution of SDH were compared between the two groups. The presence of BESS was evaluated for associations with symptoms and other CSIs.

RESULTS Of 149 patients, 43 presented with SDH-mild symptoms and 106 with SDH-severe symptoms. Patients with SDH-mild symptoms were less likely to have other CSIs (odds ratio [OR] 0.2, 95\% confidence interval [Cl] 0.08-0.5) and less likely to have severe retinal hemorrhages (OR $0.08,95 \% \mathrm{Cl} 0.03-0.3$ ). However, $60.5 \%$ of patients with SDH-mild symptoms had other CSIs. There was no difference between the groups regarding the location and distribution of SDH. Of the entire study cohort, $34(22.8 \%)$ had BESS, and BESS was present in $17(39.5 \%)$ of the SDH-mild symptoms group and $17(16 \%)$ of the SDH-severe symptoms group (OR 3.4, 95\% Cl 1.5-7.6). The presence of BESS was significantly associated with a lower chance of other CSIs (OR 0.1, 95\% CI 0.05-0.3). However, 17 patients had BESS and other CSIs. Of these 17,6 had BESS and SDH-mild symptoms.

CONCLUSIONS The high occurrence of other CSIs in patients with SDH-mild symptoms and a similar high occurrence in patients with BESS (including those with SDH-mild symptoms) indicate that such children benefit from a full evaluation for abuse.

https://thejns.org/doi/abs/10.3171/2017.7.PEDS17317

KEY WORDS child abuse; subdural hemorrhage; abusive head trauma

ABBREVIATIONS AHT = abusive head trauma; BESS = benign enlargement of the subarachnoid spaces; $\mathrm{CAP}=$ Child Abuse Pediatrics; $\mathrm{CSI}=$ concomitant suspicious injury; SDH = subdural hematoma.

SUBMITTED June 9, 2017. ACCEPTED July 18, 2017.

INCLUDE WHEN CITING Published online November 3, 2017; DOI: 10.3171/2017.7.PEDS17317. 
A BUSIVE head trauma (AHT) is the leading cause of subdural hematomas (SDHs) in young children. It has been recommended that infants with unexplained SDH be further investigated for AHT. ${ }^{2}$ Specific characteristics of SDH and head injury, such as location and number of SDHs and presence of hypoxic-ischemic encephalopathy, may be used to differentiate AHT from nonabusive injury. ${ }^{9}$ However, recent studies have furthered the understanding of $\mathrm{SDH}$ that results from nonabusive etiologies. The birthing process has been shown to cause $\mathrm{SDH}$ in approximately $40 \%$ of infants, and these SDHs have been documented to persist for up to 3 months..$^{13,18} \mathrm{~A}$ small percentage of children with benign enlargement of the subarachnoid spaces (BESS) have SDH. ${ }^{5,16,22}$ Other investigators have proposed etiologies such as sinus thrombosis, birth SDH persisting for many months beyond early infancy, and "spontaneous" as causes of SDH in young children. ${ }^{17,23}$ Although $\mathrm{SDH}$ is commonly seen in AHT, the consideration of other causes, both substantiated and hypothesized, is often necessary.

When an SDH is identified in a young child and there is no history of significant trauma, testing for occult injuries is recommended. ${ }^{2}$ Testing for such injuries is highly variable. ${ }^{12,24}$ Physicians may attribute SDH to one of these alternative causes or theories and question the utility of screening studies for occult injuries in young children with $\mathrm{SDH}$, particularly when children are clinically asymptomatic or minimally symptomatic. Minimal, transient neurological changes, such as short-lived seizures, may be attributable to the presence of SDH alone (in the absence of traumatic brain injury), as SDH has been shown to cause localized brain tissue damage capable of producing such symptoms..$^{21}$ Due to the absence of persistent neurological symptoms, physicians may be reluctant to conduct a full evaluation for abuse.

Alternative causes and theories of SDH in young children do not provide explanations for other injuries suspicious for abuse. If asymptomatic or minimally symptomatic SDH (subsequently referred to as the "SDH-mild" group) in young children is frequently caused by one or a combination of these alternatives, children with these findings should be more likely to have no other suspicious injuries than those with SDH and severe and persistent neurological symptoms ("SDH-severe" group). Additionally, if findings in the SDH-mild group are caused by something other than abuse, the location and distribution of the SDH may be different than SDH caused by abuse.

The aims of this study were to determine the prevalence of other concomitant suspicious injuries (CSIs) in young children who present with SDH-mild symptoms, and to compare that prevalence to patients with SDHsevere symptoms. Additionally, we sought to compare the locations and distributions of SDHs between the two groups. Finally, given the data supporting BESS as a potential cause of SDH in young children, we sought to evaluate the associations of BESS with SDH-mild symptoms and with CSIs.

\section{Methods}

\section{Study Design and Population}

We performed a retrospective case-control study of children younger than 2 years of age who presented to the authors' institution with SDH (as identified on a CT or MRI scan) and were evaluated by the hospital Child Abuse Pediatrics (CAP) team between June 2008 and December 2013. Cases included children with a documented history at presentation of being "asymptomatic" or "minimally symptomatic" with minimal and transient neurological symptoms that could be attributable to the presence of an SDH alone (SDH-mild group). The control group included patients with a documented history of severe and persistent neurological symptoms beginning immediately prior to identification of the SDH (SDH-severe group). An age younger than 2 years was chosen as an inclusion criteria to encompass children at the highest risk of $\mathrm{AHT}^{8}$ Children were excluded if there was a history of a preexisting abnormality of the brain, infection of the CNS, known prior intracranial hemorrhage, prior CNS surgery or shunt placement, extreme prematurity ( $<28$ weeks gestational age at birth), bone fragility syndrome, known bleeding disorder or vascular abnormality, or other condition that predisposed patients to intracranial hemorrhage, bruising, fractures, or findings that may mimic abdominal trauma, or if any of these were discovered during the medical evaluation (inclusive of the CAP team evaluation) related to the SDH. Additionally, patients with a small, isolated SDH immediately underlying a skull fracture were excluded, as these findings generally result from contact forces, are often from accidental injury, and are not the type of SDH that is explained by proven alternative causes (such as birth SDH that persist for several weeks) or theoretically explained by hypothesized causes (such as "spontaneous"). 6 The study was approved by the IRB of the authors' institution. As this was a retrospective study of de-identified data, patient informed consent was not required. This study is a secondary analysis of a subset of patients detailed previously.

\section{Data Collection}

Demographic information, past medical and clinical history, physical examination and radiological findings, and neurological outcomes were obtained through chart review. The ultimate diagnosis regarding abuse made by the CAP team was not collected and did not affect determination of whether injuries suspicious for abuse were present. Including this ultimate diagnosis as an outcome of the study would have introduced circular reasoning into the study.

\section{Study Variables}

Patients were classified as "asymptomatic," "minimally symptomatic," or "severe/persistently symptomatic" based on the definitions below. Patients were then further classified into those with and without one or more injuries suspicious for abuse (CSI) including retinal hemorrhages characteristic of abuse, retinoschisis, fractures or bruises specific for abuse, and intra-abdominal injury. The presence of any CSI in any individual patient indicated that injuries suspicious for abuse were present in that patient. Radiological imaging (MRI, CT, radiography) was performed at the discretion of the managing physicians, as was consultation of the CAP team. 


\section{Definitions}

Asymptomatic patients had a documented history of no neurological symptoms (such as vomiting, seizures, apnea, altered level of consciousness, or any other neurological symptom) and received neuroimaging (brain CT or MRI) for macrocephaly or for rapidly increasing head circumference and/or a full or tense fontanel. Minimally symptomatic patients included children who underwent neuroimaging (CT or MRI) of the brain for mild transient neurological symptoms that could be explained by the presence of SDH alone, including fussiness, vomiting, or an episode of seizure activity less than 5 minutes in length. All minimally symptomatic patients included in the study demonstrated a return to neurological baseline by the time of discharge from the hospital. Patients meeting the above definitions were combined into the SDHmild group.

SDH-severe group patients had a documented history of severe or persistent neurological symptoms including altered consciousness; persistent respiratory insufficiency and/or apnea requiring endotracheal intubation and mechanical ventilation; prolonged or recurrent seizures including status epilepticus; coma; or persistent developmental delays, without return to neurological baseline, or death. Classification of patients was performed by the study team during data abstraction. All classifications were reviewed by the study team as a whole. In cases in which all members of the study team did not agree on classification, patients were excluded from the study.

Similar to the study of McKeag et al., in this study BESS was diagnosed on neuroimaging (CT or MRI of the brain) by a pediatric neuroradiologist. ${ }^{16}$ Encephalopathy was defined as neuroradiographic findings of hypoxicischemic injury, parenchymal injury, parenchymal contusion, cerebral edema, or diffuse axonal injury, as diagnosed by pediatric radiologists based on neuroimaging. ${ }^{15}$ Patients with persistent neurological symptoms at hospital discharge included patients with persistent decrease in function with regard to consciousness (such as intractable seizures or persistent vegetative state), developmental capabilities, mobility, or bodily function (such as feeding, handling of secretions, or breathing) at the time of discharge from the $\mathrm{SDH}$-associated hospitalization.

\section{Concomitant Suspicious Injuries}

Retinal hemorrhages characteristic of abuse were defined as extensive multilayered hemorrhages extending to the ora serrata. ${ }^{11}$ Retinoschisis is the traumatic splitting of the retinal layers. ${ }^{11}$ All funduscopic examinations of patients were performed during the hospitalization related to the SDH by a pediatric ophthalmologist using indirect ophthalmoscopy and pharmacological dilation. Patients with retinoschisis and/or retinal hemorrhages characteristic of abuse were classified as having "severe retinal hemorrhages." In the study population, bruising was considered to be a CSI if any of the following were present: intraoral injury; patterned bruising; bruising in an immobile infant; bruising to the torso, eyes, cheeks, ears, neck, genitals, or buttocks; or when a caregiver disclosed abuse as the cause of the bruising. ${ }^{1,14}$ As a standard of practice, the CAP team at our institution performs complete physi- cal examinations, including documentation of any skin and intraoral injuries.

Fractures were identified on radiographs by a pediatric radiologist and were categorized as suspicious for abuse if the following were present: classic metaphyseal fractures; rib fractures; fractures of the scapula, sternum, or spinous processes; multiple fractures; fractures of different ages; fractures of the hands or feet; or complex skull fractures. ${ }^{3,10}$ Additionally, fractures in infants younger than 1 year of age without a history of trauma were considered to be suspicious for abuse. All skeletal surveys obtained conform to published recommended guidelines. ${ }^{19}$ All internal abdominal injuries were considered to be CSIs and included hollow or solid organ laceration or contusion, hollow organ perforation, and trauma to the mesentery as identified on radiographic imaging study by a pediatric radiologist or direct visualization in surgery. The necessity of further surgical and/or medical intervention for other suspicious injuries was not tracked as this would not affect study outcomes.

\section{Characteristics of SDH on Neuroimaging}

Reports from pediatric radiologists' analyses of head $\mathrm{CT}$ and/or MRI were used to classify SDH locations as unilateral or bilateral; single or multiple; convexity, supratentorial-interhemispheric, and/or infratentorial; or other.

\section{Statistical Analysis}

Statistical analyses were conducted using SPSS (version 20, IBM Corp.) and SAS (version 9.4) software. Wilcoxon rank-sum, chi-square, and Fisher exact tests were used to compare demographic variables. Chi-square and Fisher exact tests were performed to determine associations between CSIs and classification of symptoms.

Missing test data (skeletal surveys, laboratory testing for abdominal injuries, and ophthalmology examinations) were assumed to be negative for the findings of concern (fractures, intraabdominal injury, and severe retinal hemorrhages, respectively). Based on the assumption that testing rates would be lower in the SDH-mild group, this would bias the results toward validating the hypothesis that $\mathrm{SDH}$ in that group was commonly a result of the previously described alternative causes or theories (there would be fewer other suspicious injuries in the SDH-mild group).

\section{Results}

During the study period, 154 patients met initial inclusion criteria. Of those 154 patients, 5 were excluded for the following reasons: CNS infection $(n=1)$, previously known intracranial injury $(n=2)$, presence of isolated intraventricular hemorrhage $(n=1)$, and diagnosis of rickets with a small, isolated SDH immediately underlying a skull fracture $(n=1)$. Among the 149 study patients, 43 were classified as SDH-mild and 106 as SDH-severe. There were no significant differences between the two groups regarding mean age, sex, race, or insurance status (Table 1).

No significant differences in rates of obtaining skeletal surveys or ophthalmological examinations existed between the two study groups. All 149 patients had skeletal surveys performed. Of the SDH-mild group, 98\% (42 of 
TABLE 1. Characteristics of the 149 patients

\begin{tabular}{|c|c|c|c|}
\hline Characteristic & SDH-Mild (\%) & SDH-Severe (\%) & $\mathrm{p}$ Value \\
\hline No. of patients & 43 & 106 & \\
\hline \multicolumn{4}{|l|}{ Age (mos) } \\
\hline $0-2$ & $9(20.9)$ & $39(36.8)$ & \\
\hline $3-5$ & $16(37.2)$ & 34 (32.1) & \\
\hline $6-8$ & $5(11.6)$ & $18(17)$ & \\
\hline $9-11$ & $10(23.3)$ & $5(4.7)$ & \\
\hline $12-17$ & $2(4.7)$ & $7(6.6)$ & \\
\hline $18-23$ & $1(2.3)$ & $3(2.8)$ & \\
\hline Mean age (mos) & 5.9 & 4.8 & 0.06 \\
\hline Males & $30(69.8)$ & $66(62.3)$ & 0.4 \\
\hline Race & & & 0.9 \\
\hline Caucasian & 31 (72.1) & $72(67.9)$ & \\
\hline Hispanic & $2(4.7)$ & $6(5.7)$ & \\
\hline African American & $4(9.3)$ & $16(15.1)$ & \\
\hline Other & $6(14)$ & $12(11.3)$ & \\
\hline Payor & & & 0.1 \\
\hline Private & $10(23.3)$ & $12(11.3)$ & \\
\hline Medicaid & $28(65.1)$ & $80(75.5)$ & \\
\hline Uninsured/other & 0 & $5(4.7)$ & \\
\hline Military & $5(11.6)$ & $9(8.5)$ & \\
\hline
\end{tabular}

All data given as number of patients (\%) unless otherwise indicated.

43) had retinal examinations, whereas $100 \%$ of the SDHsevere group had such examinations. Regarding laboratory screening for abdominal trauma (liver function tests or pancreatic enzymes), $84 \%$ of the SDH-mild group was screened, compared with $97 \%$ of the SDH-severe group (OR 0.1, 95\% CI 0.04-0.6). The SDH-mild group was less likely to undergo an abdominal CT scan (16\% vs $47 \%$, OR $0.2,95 \%$ CI $0.09-0.5)$. As previously described, all missing data (lack of tests) were assumed to be negative test results, thus biasing the results toward the hypothesis that SDH in the SDH-mild group is caused by something other than abuse.

Table 2 compares the prevalence of CSI between the two groups. Patients in the SDH-mild group were significantly less likely to have 1 or more CSIs (OR $0.2,95 \%$ CI $0.08-0.5)$. Another way of representing this finding is that patients in the SDH-severe group were 5 times more likely to have a CSI. Despite the significant difference between the groups, $60.5 \%$ of patients in the SDH-mild group had a CSI. Of the 23 patients in the SDH-mild group who did not have bruises (and thus, their suspicious findings, if present, would only be identified by investigation beyond a complete physical examination), 6 (26\%) had a CSI. Of these 6 patients, 2 had retinal hemorrhages characteristic of abuse and 4 had fractures, 3 of whom had multiple extremity and/or rib fractures. The SDH-mild group was also significantly less likely to have 3 or more categories of CSIs and less likely to have severe retinal hemorrhages. Again, these findings may also be represented by stating the SDH-severe group was 15 times and 24 times more likely to have 3 or more categories of CSI and severe retinal hemorrhages, respectively. No difference existed be- tween the groups with respect to prevalence of suspicious bruising, fractures, or internal abdominal injury. Of note, 11 patients in the SDH-mild group had no symptoms, and of this subset, 3 (27\%) had CSI.

The characteristics of intracranial hemorrhage in the study patients are described in Table 3 . No significant differences existed between the study groups. Of note, nearly all patients had SDH over the convexities, a finding that has previously been associated with abuse. ${ }^{9}$ Additionally, approximately one-fifth of SDH-mild patients had parenchymal hemorrhage, which is not explained by any of the alternative causes or theories.

Of the entire study cohort, 34 (22.8\%) had BESS, and BESS was present in 17 (39.5\%) of the SDH-mild group and $17(16 \%)$ of the SDH-severe group (OR 3.4, 95\% CI 1.5-7.6). The presence of BESS was significantly associated with a lower occurrence of a CSI (OR 0.1,95\% CI 0.05-0.3). However, 17 patients had BESS and a CSI. Of these 17 patients, 6 had BESS and SDH-mild symptoms. Thus, more than one-third of the patients with BESS and SDH-mild symptoms had CSI.

\section{Discussion}

The identification of an SDH in a young child in the absence of a known history of severe trauma often triggers a child abuse evaluation. However, due to published peer-reviewed studies, some nontraumatic causes of SDH are gaining acceptance as plausible causes in otherwise apparently well children. Other causes are simply hypothesized, with no or minimal supporting evidence. Given the perceived social risks associated with an abuse evaluation, and the risks of additional radiation from screening tests for abuse, physicians may opt out of conducting an abuse evaluation in children with SDH and no or minimal neurological symptoms. This study identified other suspicious injuries in more than $60 \%$ of patients with no or minimal neurological symptoms, including fractures and severe retinal hemorrhages. Importantly, the study design biased results toward a lower rate of identification of suspicious injuries in the group with no or minimal symptoms. The study patients did not include young children with SDH who were not referred for a CAP evaluation. However, the very high percentage of SDH-mild patients with other suspicious findings raises concern that many young children with SDH and no or minimal symptoms who are not referred for a CAP evaluation may also have a CSI that, absent such an evaluation, remains undetected, placing these children at great risk of further injury or death. ${ }^{20}$ Given the high occurrence of identification of CSI in young children with SDH and no or minimal symptoms, a full evaluation for abuse is frequently warranted in this group.

Despite the high prevalence of CSIs in patients in the $\mathrm{SDH}$-mild group, patients in the SDH-severe group were much more likely to have a CSI. These findings may be interpreted in several ways. Patients with SDH and severe symptoms may simply have been abused in a more severe manner. Conversely, a subset of the patients in the SDHmild group and no CSI could, potentially, have an SDH due to a nonabusive etiology. This study does not completely refute or confirm the validity of nonabusive causes 
TABLE 2. Concomitant suspicious injuries in the study patients

\begin{tabular}{lccc}
\hline \multicolumn{1}{c}{ CSIs } & SDH-Mild (\%) & SDH-Severe (\%) & OR (95\% CI) \\
\hline Total no. of patients & 43 & 106 & \\
\hline No. of patients w/ 1 or more suspicious injuries & $26(60.5)$ & $94(88.7)$ & $0.2(0.08-0.5)$ \\
\hline 1 category of injury present* ${ }^{*}$ & $12(27.9)$ & $34(32.1)$ & $0.8(0.4-1.8)$ \\
\hline 2 categories of injury present & $13(30.2)$ & $28(26.4)$ & $1(0.5-2.2)$ \\
\hline 3 or more categories of injury present ${ }^{*}$ & $1(2.3)$ & $58(54.7)$ & $0.07(0.009-0.5)$ \\
\hline Severe retinal hemorrhagest & $4(9.3)$ & $83(78.3)$ & $0.08(0.03-0.3)$ \\
\hline Any & $15(34.9)$ & $52(49.1)$ & $0.1(0.07-0.3)$ \\
\hline Characteristic of abuse & $3(7)$ & $23(21.7)$ & $0.08(0.02-0.3)$ \\
\hline Retinoschisis & $1(2.3)$ & $58(54.7)$ & $0.09(0.01-0.7)$ \\
\hline Fractures & $16(37.2)$ & $18(17)$ & $0.5(0.2-1)$ \\
\hline Single & $5(11.6)$ & $40(37.7)$ & $0.6(0.2-1.9)$ \\
\hline Multiple & $11(25.6)$ & $56(52.8)$ & $0.6(0.3-1.2)$ \\
\hline Bruises & $20(46.5)$ & $13(12.3)$ & $0.8(0.4-1.6)$ \\
\hline Internal abdominal injury & $1(2.3)$ & $0.2(0.02-1.3)$ \\
\hline
\end{tabular}

* Individual categories include retinal hemorrhages characteristic of abuse/retinoschisis, fractures, bruising, and internal abdominal injury.

$\dagger$ Only patients with retinal hemorrhages that were characteristic of abuse and/or retinoschisis were classified as having severe retinal hemorrhages. Patients with both retinoschisis and retinal hemorrhages characteristic of abuse were counted only once when tabulating "severe retinal hemorrhages." Six patients in the SDH-severe cohort had retinoschisis but did not have other retinal hemorrhages characteristic of abuse, resulting in a total of 58 patients with severe retinal hemorrhages.

of SDH in young children. It does, however, support the need for screening for other suspicious injuries in most, if not all, young children with SDH and no obvious traumatic mechanism, regardless of the presence of BESS or absence of severe or persistent neurological symptoms.

A number of recent studies have supported BESS as a possible cause of SDH..$^{5,16,22}$ In this study, the presence of BESS significantly decreased the likelihood of identification of other suspicious injuries, supporting BESS as a possible cause of SDH. Conversely, of the 34 patients in this study with BESS, half of them had CSI, and more than onethird of those with BESS and SDH with mild symptoms had CSI. Physicians who encounter a young child with BESS and SDH should not reflexively determine that BESS is the cause of the SDH and then forgo an abuse evaluation.

Multiple, interhemispheric, and convexity SDHs have been associated with abuse. ${ }^{9}$ In this study, there were no statistical differences in SDH locations/characteristics between patients with and without severe and/or persistent neurological symptoms. Given the prior data from a large systematic review supporting the association between multiple, interhemispheric, and convexity SDH and abuse, ${ }^{9}$ it is likely that many of the children in this study with such SDHs were abused, but had no or minimal symptoms at the time of presentation and/or had no CSIs. It is also possible that the sample size was not large enough to detect a difference between the two groups.

When evaluating the findings of this study as a whole, the lower occurrence of CSIs in patients in the SDH-mild group compared with those in the SDH-severe group supports the hypothesis that $\mathrm{SDH}$ in the first group may be caused by something other than abuse. These findings must be interpreted in the context of the study's purposeful bias in the direction of this hypothesis. However, the lack of difference in location and characteristics of SDH between the two groups argues against this hypothesis. As such, the results of this study with respect to the hypotheses are mixed. It is possible that a small number of patients in the SDH-mild group had SDH due to an alternative (nonabusive) etiology, and this small number was not large enough to be reflected in the comparison of neuroradiographic findings. Alternatively, the mixed results may be due to a number of the patients in the SDH-mild group suffering abuse in a less severe manner, causing fewer CSIs. In either case, this study does not support assertions that a very large number of children have SDH caused by alternative or hypothesized causes. ${ }^{4}$ BESS was associated with fewer CSIs, but half of the patients with BESS had a CSI. The high occurrence of CSIs in patients in the SDHmild group and a similar high occurrence in patients with BESS (including those in the SDH-mild group) indicate that such children benefit from a full evaluation for abuse, including a complete physical examination, skeletal sur-

TABLE 3. Neuroradiographic findings in the study patients

\begin{tabular}{lccc}
\hline \multicolumn{1}{c}{ Finding } & $\begin{array}{c}\text { SDH-Mild } \\
(\mathrm{n}=43)\end{array}$ & $\begin{array}{c}\text { SDH-Severe } \\
(\mathrm{n}=106)\end{array}$ & $\begin{array}{c}\text { OR } \\
(95 \% \mathrm{Cl})\end{array}$ \\
\hline SDH characteristic & & & \\
\hline Unilateral & $12(27.9)$ & $28(26.4)$ & $1.1(0.5-2.4)$ \\
\hline Bilateral & $31(72.1)$ & $78(73.6)$ & $0.9(0.4-2.1)$ \\
\hline Multiple & $38(88.4)$ & $98(92.5)$ & $0.6(0.2-2)$ \\
\hline Convexity & $43(100)$ & $103(97.2)$ & $\mathrm{NA}$ \\
\hline Interhemispheric & $18(41.9)$ & $63(59.4)$ & $0.5(0.2-1)$ \\
\hline Posterior fossa & $12(27.9)$ & $38(35.8)$ & $0.7(0.3-1.5)$ \\
\hline SAH (any) & $9(20.9)$ & $37(34.9)$ & $0.5(0.2-1.1)$ \\
\hline Parenchymal hemorrhage & $8(18.6)$ & $31(29.2)$ & $0.5(0.2-1.3)$ \\
\hline
\end{tabular}

$\mathrm{NA}=$ not applicable; $\mathrm{SAH}=$ subarachnoid hemorrhage.

All data given as number of patients (\%) unless otherwise indicated. 
vey, ophthalmology examination, and laboratory screening for abdominal trauma.

This study has several limitations. This was a singleinstitution, retrospective study. Different results may have been achieved in a different location, but it is unlikely that SDH characteristics and symptomatology vary based on geography. The study patients were all referred for child abuse pediatric consultations by managing physicians. There may have been other patients with SDH, in either study group, who were not seen by CAP, as no standard process for evaluation of all SDHs existed during the study period. However, the high occurrence of CSIs in the SDHmild group suggests that most, if not all, young patients with such findings necessitate an abuse evaluation, including children with BESS. This study did not include neck injuries as a suspicious finding, as neck CT or MRI did not become standard practice in cases of AHT until after the study data collection was completed.

\section{Conclusions}

The lower prevalence of other suspicious findings in young children in the SDH-mild group may indicate that some SDHs in this group were caused by conditions other than abuse. Conversely, some of these patients may have been abused in a less severe fashion. The high occurrence of CSIs in patients in the SDH-mild group and a similar high occurrence in patients with BESS (including those in the SDH-mild group) indicate that such children benefit from a full evaluation for abuse. Future prospective studies with a standardized evaluation for all young children with SDH may be beneficial in further clarifying the utility of screening for other suspicious injuries and underlying etiology of SDH.

\section{References}

1. Anderst JD, Carpenter SL, Abshire TC: Evaluation for bleeding disorders in suspected child abuse. Pediatrics 131:e1314-e1322, 2013

2. Christian CW: The evaluation of suspected child physical abuse. Pediatrics 135:e1337-e1354, 2015

3. Cooperman DR, Merten DF: Skeletal manifestations of child abuse, in Reece RM, Christian CW (eds): Child Abuse Medical Diagnosis and Management, ed 3. Elk Grove Village, IL: American Academy of Pediatrics, 2009, pp 121-165

4. Gabaeff SC: Investigating the possibility and probability of perinatal subdural hematoma progressing to chronic subdural hematoma, with and without complications, in neonates, and its potential relationship to the misdiagnosis of abusive head trauma. Leg Med (Tokyo) 15:177-192, 2013

5. Greiner MV, Richards TJ, Care MM, Leach JL: Prevalence of subdural collections in children with macrocrania. AJNR Am J Neuroradiol 34:2373-2378, 2013

6. Gulino SP: Autopsy findings, in Frasier L, Rauth-Farley K, Alexander R, Parrish R (eds): Abusive Head Trauma in Infants and Children: A Medical, Legal, and Forensic Reference. St. Louis: GW Medical Publishing, 2006, pp 297-313

7. Hansen JB, Frazier T, Moffatt M, Zinkus T, Anderst JD: Evaluation of the hypothesis that choking/ALTE may mimic abusive head trauma. Acad Pediatr 17:362-367, 2017

8. Keenan HT: Epidemiology of abusive head trauma, in Jenny C (ed): Child Abuse and Neglect: Diagnosis, Treatment, and Evidence. St. Louis: Saunders Elsevier, 2011, pp 35-38
9. Kemp AM, Jaspan T, Griffiths J, Stoodley N, Mann MK, Tempest V, et al: Neuroimaging: what neuroradiological features distinguish abusive from non-abusive head trauma? A systematic review. Arch Dis Child 96:1103-1112, 2011

10. Kleinman PK, Rosenberg AE, Tsai A: Skeletal trauma: general considerations, in Kleinman PK (ed): Diagnostic Imaging of Child Abuse, ed 3. Cambridge, UK: Cambridge University Press, 2015, pp 23-52

11. Levin AV: Retinal hemorrhages: advances in understanding. Pediatr Clin North Am 56:333-344, 2009

12. Lindberg DM, Beaty B, Juarez-Colunga E, Wood JN, Runyan DK: Testing for abuse in children with sentinel injuries. Pediatrics 136:831-838, 2015

13. Looney CB, Smith JK, Merck LH, Wolfe HM, Chescheir NC, Hamer RM, et al: Intracranial hemorrhage in asymptomatic neonates: prevalence on MR images and relationship to obstetric and neonatal risk factors. Radiology 242:535-541, 2007

14. Maguire S, Mann M: Systematic reviews of bruising in relation to child abuse-what have we learnt: an overview of review updates. Evid Based Child Health 8:255-263, 2013

15. Maguire S, Pickerd N, Farewell D, Mann M, Tempest V, Kemp AM: Which clinical features distinguish inflicted from non-inflicted brain injury? A systematic review. Arch Dis Child 94:860-867, 2009

16. McKeag H, Christian CW, Rubin D, Daymont C, Pollock AN, Wood J: Subdural hemorrhage in pediatric patients with enlargement of the subarachnoid spaces. J Neurosurg Pediatr 11:438-444, 2013

17. Miller D, Barnes P, Miller M: The significance of macrocephaly or enlarging head circumference in infants with the triad: further evidence of mimics of shaken baby syndrome. Am J Forensic Med Pathol 36:111-120, 2015

18. Rooks VJ, Eaton JP, Ruess L, Petermann GW, Keck-Wherley J, Pedersen RC: Prevalence and evolution of intracranial hemorrhage in asymptomatic term infants. AJNR Am J Neuroradiol 29:1082-1089, 2008

19. Section on Radiology: Diagnostic imaging of child abuse. Pediatrics 123:1430-1435, 2009

20. Sheets LK, Leach ME, Koszewski IJ, Lessmeier AM, Nugent M, Simpson P: Sentinel injuries in infants evaluated for child physical abuse. Pediatrics 131:701-707, 2013

21. Squier W, Mack J, Green A, Aziz T: The pathophysiology of brain swelling associated with subdural hemorrhage: the role of the trigeminovascular system. Childs Nerv Syst 28:20052015,2012

22. Tucker J, Choudhary AK, Piatt J: Macrocephaly in infancy: benign enlargement of the subarachnoid spaces and subdural collections. J Neurosurg Pediatr 18:16-20, 2016

23. Vinchon M, Delestret I, DeFoort-Dhellemmes S, Desurmont M, Noulé N: Subdural hematoma in infants: can it occur spontaneously? Data from a prospective series and critical review of the literature. Childs Nerv Syst 26:1195-1205, 2010

24. Wood JN, Feudtner C, Medina SP, Luan X, Localio R, Rubin DM: Variation in occult injury screening for children with suspected abuse in selected US children's hospitals. Pediatrics 130:853-860, 2012

\section{Disclosures}

Dr. Anderst reports that he has served as a consultant to the prosecution and defense in cases of alleged child abuse.

\section{Author Contributions}

Conception and design: all authors. Acquisition of data: Hansen. Analysis and interpretation of data: Anderst, Hansen, Frazier, Moffatt. Drafting the article: Anderst, Hansen. Critically revising 
the article: Anderst, Frazier, Moffatt, Zinkus. Reviewed submitted version of manuscript: all authors. Approved the final version of the manuscript on behalf of all authors: Anderst. Statistical analysis: Anderst, Hansen. Study supervision: Anderst.

\section{Supplemental Information}

Previous Presentations

Portions of this manuscript were presented in oral form at the
Ray Helfer Society Annual Meeting, Denver, Colorado, April 3, 2017, and again in oral form at the Pediatric Academic Societies Annual Meeting, San Francisco, California, May 8, 2017.

\section{Correspondence}

James Anderst, Department of Pediatrics, Children's Mercy Hospital, 2401 Gillham Rd., Kansas City, MO 64108. email: jdanderst@cmh.edu. 